\title{
Study of Mechanical Performance Affecting Factors in Split Casing Pump
}

\author{
Ramesh. S. Gavade, Suresh M. Sawant
}

\begin{abstract}
The need for better performance and higher comfort continually drive search for new features. In today's applications, pumps are expected to continue continuously for a long time. So "operational reliability" is a key factor. The comprifugal pump is a fluid-carrying machine, with centrifugal energy produced by rotating air. Pumps are used for conveying fluids. Rotating power usually comes from an electric car, which is turned by the engine and the engine. There is a great demand for centrifugal pumps on the market. The construction is relatively inexpensive, durable and easy, and its high speed makes it possible to connect the pump directly to an unmatched car. The centrifugal pump provides a constant flow of fluid, and can be easily blown without causing damage to the pump. Pump reliability problems address a large amount of repair repairs and the cost of loss of access to chemical plants, refineries, and many electrical appliances. This paper describes the most common causes of pump failure, and how they can be avoided in most cases by using the appropriate types of analysis and procedure during the pump selection process. Specifically, key issues include where the pump will operate next to the best efficient point (BEP). Proper pump selection and installation avoid misalignment. This paper explains the various reasons for the failure of Split break pumps.
\end{abstract}

Key Words: BEP, split pump, centrifugal pump, gland, vibration.

\section{INTRODUCTION}

Centrifugal pump is a hydrodynamic pump, in which the rotating impeller continuously transfers the mechanical work of the machine from the drive to the liquid. The kinetic energy is converted into potential pressure potential. Centrifugal pumps are usually pieces of the most reliable equipment. Pump manufacturers and suppliers, such as carry-off manufacturers; spend a lot of research money to continuously improve the performance and reliability of their equipment. Industrial pump operators have also contributed to this increase in pump technology, by combating problems associated with certain complex systems, especially applications that require significant variations in load or temperature. Where space constraints do not allow sufficient information to be provided to a student to access or use the specified technology, references that provide additional information will be provided.

Revised Manuscript Received on November 25, 2020.

* Correspondence Author

Ramesh.S.Gavade*, Research Scholar, Mechanical Engineering RIT Rajaramnagar, Shivaji University India, Email: rameshaditiom21@gmail.com

Suresh M. Sawant, Mechanical Engineering RIT, Rajaramnagar, Shivaji University India, Email: suresh.sawant@ritindia.edu

(C) The Authors. Published by Blue Eyes Intelligence Engineering and Sciences Publication (BEIESP). This is an open access article under the CC BY-NC-ND license (http://creativecommons.org/licenses/by-nc$\mathrm{nd} / 4.0 /$ ) fractures, fatigue, aging, or leaks. Nourbakhsh et.al [1] showed that fractures occur as a result of overloading, for example from higher pressure than expected or loading of the pipe above the recommended levels. Chan et.al. [2] showed the fatigue requires that the loads be loosened so that the stress exceeds the tolerance limit. fatigues in the pump parts is frequently brought about by inordinate vibration, which additionally makes the rotor sneak out of equilibrium, within the sight of extreme misalignment between the pump and shaft drive zones, unreasonable pass throbs, or unnecessary development intensified by regular recurrence reverberation. Veness et.al.[3] proved that More than $80 \%$ of all issues fall into the classifications recorded previously. There are sure methodologies and techniques that can be followed that lessen the opportunity of encountering such issues, or that help decide how such issues can be tackled on the off chance that they happen. Lorenc et.al.[4] studied that centrifugal pumps, split casing pumps are the most popular model in the market, used for a variety of applications such as water works, small and large irrigation schemes, cooling towers, cooling air, water distribution, water spraying systems. These are also used in the industries of paper, sugar, textiles, where plants produce less destructive liquids and turbid water with special building materials, in critical power application stations, in contaminated water etc [5]. A separate case pump is defined as a "Centrifugal pump where a pump cylinder is made up of two pieces that are joined together by bolts". The cake separating pumps can be split vertically (unlike shaft direction), or horizontally separated (similar to shaft direction). SCT pumps are usually recommended for clear, cold water [6]. These pumps have keys separated in half by means of a drill design, impeller enclosure. The biggest advantage of the size separation pump is that the internal pump can be tested by removing only the upper case; no need to remove its Rotor.

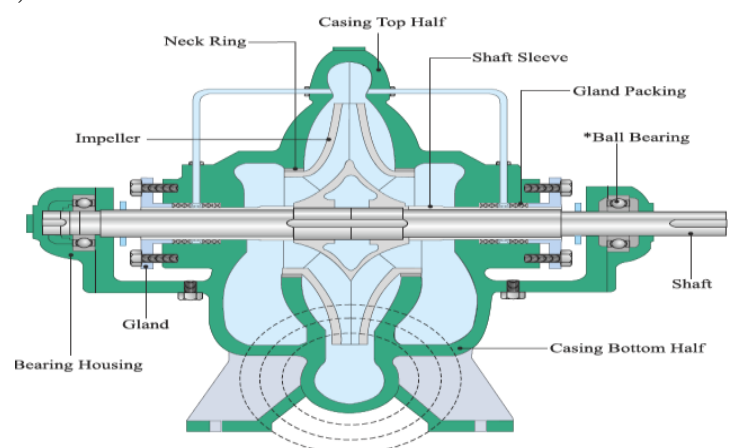

Fig 1 - Split Casing pump

II. SHAFT SEALING:

Published By:

Blue Eyes Intelligence Engineering and Sciences Publication

(C) Copyright: All rights reserved.

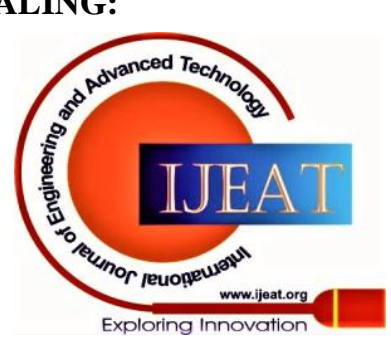




\section{Study of Mechanical Performance Affecting Factors in Split Casing Pump}

Almost everywhere, where pumps with rotating shafts are used. The shaft seal creates a barrier between the inside of the pump and the air. The pump with the through-shaft is not completely shut off.

It is a challenge for the entire pump industry to reduce leaks. Seal performance greatly influences pump performance. When properly operated, the signal is not detected [7]. As soon as it starts to leak, however, serious problems can arise, either with the pump or with the environment. The value of the shaft cover should not be underestimated during pump design, operation, or repair.

\section{II-a) Shaft sealing by Gland packing}

Conventional shaft sealing - Fig 4 shows a marking on the insertion of the gland where the packing rings are designed as controlled leakage devices and should be allowed to leak in a series of 10 to 20 drops per minute to keep them moisturized and reduce wear or damage wear. Hunger is used to keep pressure on the packaging rings to mark the right. Lantern ring is used to form a water film, in which to push the box having a lower pressure than the atmospheric pressure \& used to maintain lubrication to packing hunger. The proposed poverty line for pump applications is now rarely installed in new pumps. This is due to its high storage requirements. Packing hunger requires regular checks and maintenance adjustments. Proper lubrication of the hunger pack requires a certain amount of leakage [8]. Severe fluid conditions (organisms, additives, overheating) can significantly reduce their service life. Packed glands should be used in conjunction with the shaft sleeves to avoid damage to the shaft of the aggressive liquid or as a result of improper treatment of the full gland. The use of gland packing shaft closure will reduce costs but is a source of contamination, asbestos is restricted, excessive collisions leading to increased heat production, low efficiency, low lifespan due to water intrusion on bearings, high storage costs, time remaining and energy consumption a lot.

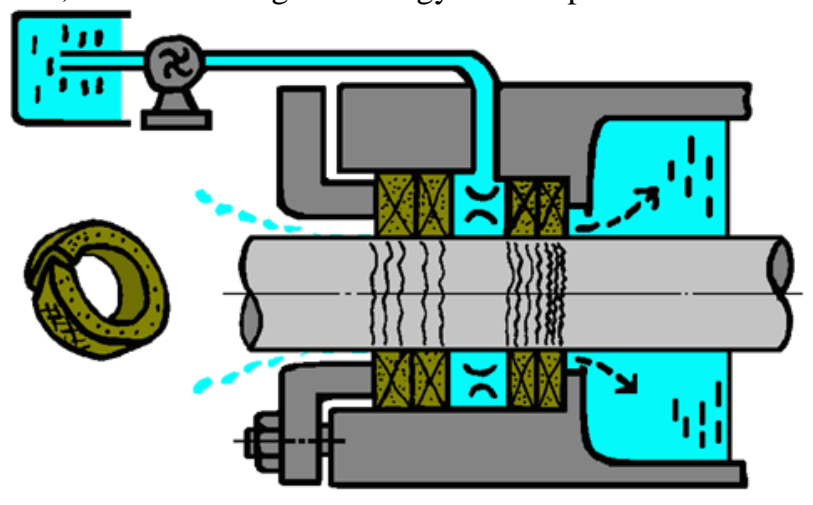

Fig 2 - Sealing by Gland Packing

\section{II-b) Shaft sealing by mechanical seal}

Equipment markers, number 5, are widely used in industrial pumps and can provide good health. The mechanical shaft seal is actually a throttle mounted around the shaft. Reduce leakage between the pump and the surrounding area to a minimum. The opening between the vertical and rotating part of the sign should be small to minimize leakage. They too, however, are controlled leak devices. Marked surfaces are not allowed to communicate in a dry area. Some of the liquid should pass between the rotating surface and the surface to reduce friction and remove heat generated. Seals and two seals, as well as gas-purified seals, have been used in complex sealing services. These programs can be very large and expensive [9]. Mechanical shaft signal failure is the most common cause of pump failure. The shaft seal is exposed to very different operating conditions. Sometimes the working conditions change so that they are very different from the specific conditions for which the mark was intended [10]. The failure of the Mechanical Seals appears to decrease in four broad stages - the movement of the seal is restricted and the surface is opened, the temperature causes 0 rings to drop, the building materials are attacked by a closed liquid. \& seal is incorrectly installed [11]. High efficiency and costly care is a requirement associated with the use of pumps for these shaft lock systems.

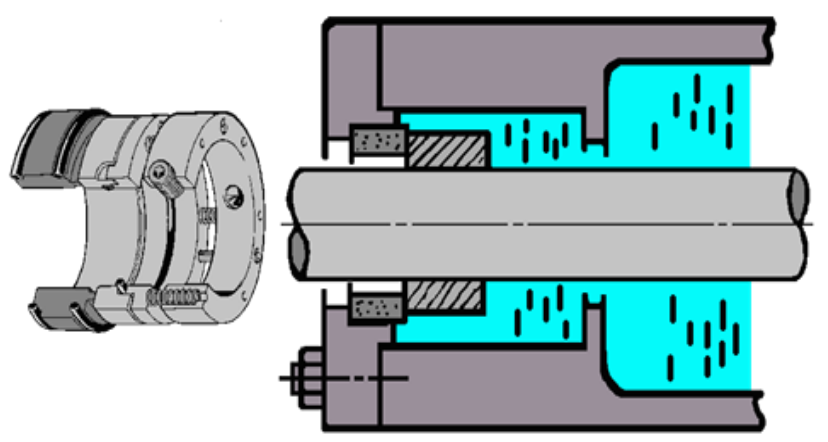

Fig 3 - Sealing by Mechanical Seal

\section{FAILURE OF ANTIFRICTION BALL BEARING}

The purpose for the metal roller is to decrease rotational grinding and to help outspread and hub loads. It accomplishes this, by utilizing at any rate two competitions to contain the balls and move the heaps to the balls. In many frameworks, one race is halted and the other is connected to a turning get together (e.g., harp or shaft). As one of the races hefts around it makes the balls turn too [12]. It only connects to the indoor and outdoor race in a very small area, which helps it to rotate very smoothly. But it also means that there is no contact area holding that load, so if the load is overcrowded, the balls can cripple or explode, damaging the load. Please note, when the pump operates in the area of maximum efficiency (BEP) the only load the ball has to carry, the weight of the rotating assembly, the pressure caused by the equal distortion of the shaft [13]. If you think the application is right for the first time, the berets should be properly fitted, oiled and maintained. Poor workplaces, especially wet or polluted areas and poor management practices invite early failure. When the bearing fails, it is important to find the exact cause so that appropriate adjustments can be made. Failure mode tests often reveal the real cause of the failure. This process is so complex that one failure mode can start another. The fact is that many bearings are overcrowded due to, improper balance between the bearing and the shaft (the shaft was unbearable), misalignment between the pump and its driver, folding hole, uneven rotating object, far from the blown arm, pump performance away from its efficient point severe (BEP), cooling the outer scalp causes it to shrink, increase distortion and cause more stress. Cavitation, water hammer, axial focus,

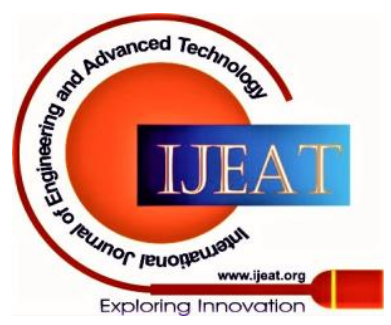


carrying housing sometimes comes out all around, impeller is located too far away and carrying is one of the reasons for failure to carry [14].

Bears are the main support for the rotor system, and they carry loads carried by the operation of impellers and other liquid components in the Rotor. These incorporate, yet are not restricted to, weight, irregularity, unsteadiness, and volute or diffuser stacking as an afterthought and hub push. Bears can be a liquid film or a moving component (likewise called antifriction) [15]. Liquid film orientation incorporate modest however precarious heading, and bore contored (to reinforce and/or settling) bears, for example, pressure dam or multiaxial groove course, and inclining cushion direction. Moving component orientation incorporate roller barrel shaped heading (convey a substantial burden yet a little pivotal burden), metal rollers (particularly for outspread restricted, yet in various organization designs can take a weighty charge for overlooking a specific measure of diffuse burden)[16]. Studies have indicated that course are immediate or backhanded (e.g., due to insufficient signal failure or insufficiency) which accounts for about half of all pump closures prematurely.

\section{CASING}

The case converts the velocity force into a compressive force. Casing is divided axially into two parts, cable above and below. The size of the casing below has interlocking feet which reduces the chances of vibration.

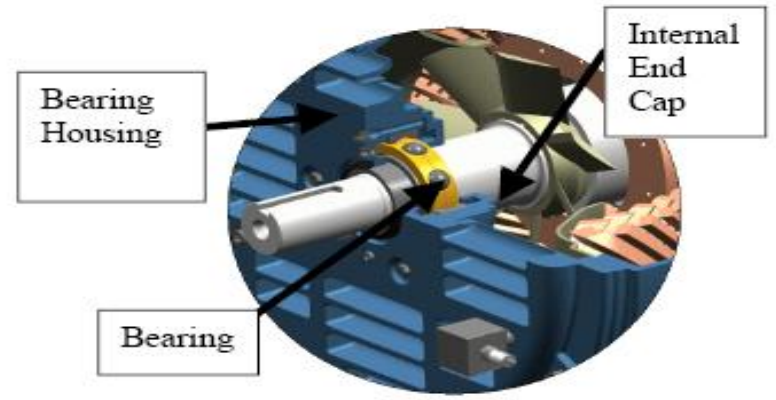

Fig 4 - Anti-friction Bearing Arrangement

A pump cone is known as a casing or lodging in certain sorts of gear. Its motivation is to give last, ground-based, rotating, and give a very much depleted progression of pumped fluid. Housings can be made of an assortment of materials, including cast-iron, copper, carbon steel and hardened steel. Particularly on enormous pumps, housings are quite often disseminated consistently. The chose thing relies upon its quality, cost, and protection from consumption, just as the harming highlights of its erosion opposition. The principle mechanical issues in the housings are their protection from obstruction and weight under tension and stacking of the spout, the propensity of multistage housings to take a "bow" on the inner endorsement request on the off chance that it is warmed excessively quick or if the finish of the pump isn't allowed to grow, spilling gaskets or gaskets spine jolts are isolated, with trouble of specific kinds of housings to dodge loss of interior gasket preload, and inside harm identified with the fence harm. Regarding the last two issues, the difference in characteristic causes a few years back from asbestos to vegetable fiber and other delicate gaskets has prompted the spillage of spine seal and stomach seal victories where it was not already. Carbon fiber gaskets were found to have a "spring back" toughness after starting asbestos-like weight, and is suggested in these cases. Furthermore, elastic, metal gaskets are likewise better alternatives.

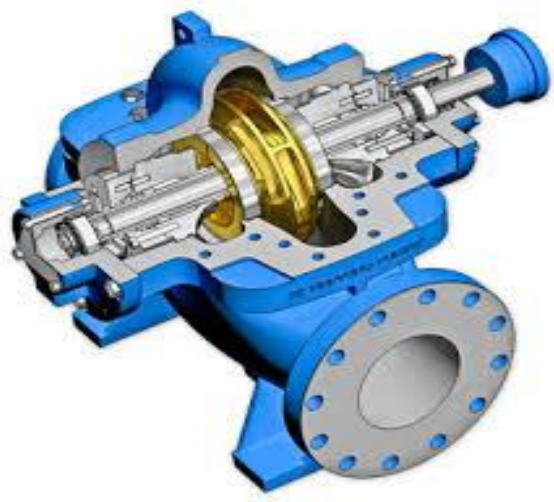

Fig 5 - Lower \& upper half casing

\section{IMPELLER}

The impeller is the main source of energy for the pump flow. Double-drag geometry reduces axial focus and improved compression conditions. In a centrifugal pump, the impeller takes the axial flow to the pump entry point, and turns its direction in a more or less direct direction, simultaneously increasing the flow head due to the centrifugal force field to pass through it. Axial flow and mixed flow pumps form a small head at each stage, but are usually able to control the maximum flow of a given ammunition range, and provide all or most of the flow energy due to the lifting effect inside the pump pumps, acting as airfoils as in an airplane. Similar sorts of building materials are frequently used to introduce articles, for example, housings, and impellers are regularly (however not generally) disposed of because of the ease of large scale manufacturing. It is significant in the choice of the pump and the establishment to make the trial of the incense head and stream in examination with what was really made. Choose a pump that will work close to the most efficient pump point. Contrary to intuition, centrifugal pumps do not receive small loading and vibration as they are pushed back, unless constipation is achieved with variable speed operation. Performance under BEP at any given speed, such as performance beyond that point, results in inconsistencies in the flow of flow in the aircraft and in diffuser vanes or in languages of various categories. This loads the bakkies, and can lead to "flight reduction," and the corresponding production of solid vortices can severely disrupt the entire rotor system, and can lead to fatigue of fabrics or distribution plates.

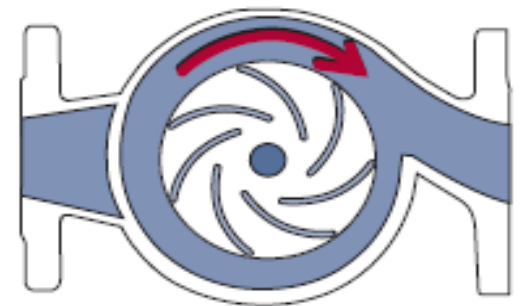

Fig 6 - Liquid Flow through impeller

Published By:

Blue Eyes Intelligence Engineering and Sciences Publication

(C) Copyright: All rights reserved.

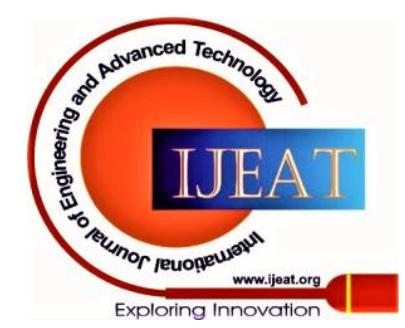




\section{Study of Mechanical Performance Affecting Factors in Split Casing Pump}

Many plants purchase more powerful equipment than is needed, allowing for future product growth, but doing so ensures years of unreliable operation of potentially reliable equipment. Never use the pump for long periods of time in the flow below the "continuous flow" provided by the manufacturer.

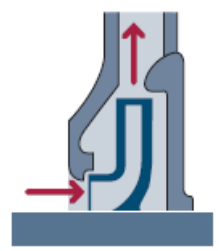

Radial flow pump
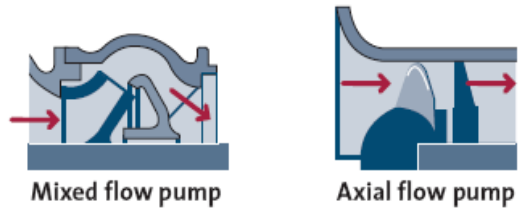

Axial flow pump

Fig 7 - Different types of pumps

The pump suction configuration has significant mechanical properties. Both the association of the rib pumping gear, just as the water driven development over the pump pressure are significant in such manner. A significant issue with water driven concerns is that adequate attractions pressure is available to forestall Cavitation. Today, it is justifiable that this implies something other than having enough NPSHA to fulfill the three percent decrease in NPSHR distributed by the producer. At NPSHA, around 3 NPSHR early Cavitation can cause serious disintegration on the tow zone or wear rings. Regardless of whether the NPSHA is sufficiently high to forestall Cavitation under typical conditions, it can at present be caused in neighbourhoods a contorted or turning delta pipe, as it tends to be the aftereffect of an elbow nearest to the pump pull banner, too close an incline close to the attractions fold, or vortices at the channel. On the off chance that the pump is worked excessively far away from its BEP, the point of approaching stream into the pivoting impeller valve may vary from the desires for the pump producer at that pump speed, and the suspension of the vane may either suck or release, bringing about a draw or re-release, separately. Redundancy of such interior stream can cause Cavitation on the weight side of the bakkie, and can make storm-like swirls surrounding the plane, however at a more slow, additionally energizing rotor at basic paces at startling frequencies.

\section{WEAR RING}

A wear ring is used to store the permit between the impeller \& casing. A small removal improves the efficiency of the pump as the leakage is reduced from high pressure (on the delivery side) to low pressure side (suction side). Wear rings, in some cases called incense neck rings, are annular tube shaped regions that structure a tight hole between the "circular" texture of the incense and the encompassing bit of packaging. Their principle reason for existing is to give a non-detached sign that forestalls the release of liquid close to the source from the release back to the low-pressure drop. The second capacity of the wear rings is to give hydrostatic rotor uphold utilizing an article known as the "Lomakin Effect," which permits the wear rings not exclusively to fill in as images yet additionally as fragile bears yet deliberately positioned. The impact of Lomakin can prompt the reiteration of the primary basic speed at high warmth pumps. High basic rates are additionally influenced, however typically lower. A full conversation of Lomakin's effect is given in the vibration observing area

Published By:

Blue Eyes Intelligence Engineering and Sciences Publication (C) Copyriaht: All riahts reserved. underneath. Wearing rings can be plain (i.e., smooth surface) or scored. Striped wear rings are less inclined to permitting particles in the skin to cause rotor breakdown, and furthermore give a superior fixing capacity. Notwithstanding, broke rings have a slight Lomakin impact. On the off chance that the furrows are profound or wide enough (the request is more than 0.150 inches), they may wind up with a Lomakin impact. Also, as wear rings increase their radial exposure due to aging or erosion, Lomakin effect is lost at a rate commensurate with the increase in clearance. This can cause the critical rotor speed to drop as the components age, possibly causing unexpected high vibrations at some point before the pump is often repaired due to a decrease in hydraulic performance.

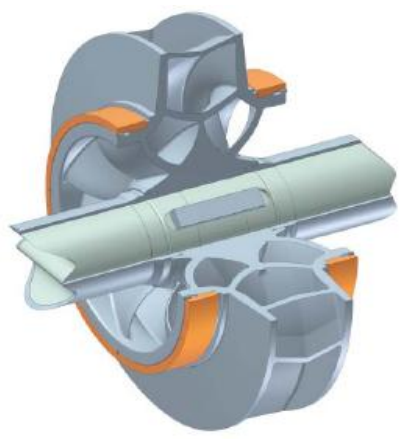

Fig 8 - Wear Ring

\section{BEARING HOUSING}

Indoor installation provides structural support for bears, and their durability should be indicated by the series and the strength of the bearing in determining the amount of active rigidity provided by the rotor. Providing housing provides convenient driving and endless driving experience. Typically, non-motorized driving ends are found to take axial \& radial load, due to the same life of NDE on the lower side and DE side bearing takes on a hot increase and takes only the spreading load. Carrying life of DE side bearing compared to the upper side.The most wellknown vibration issues with level pumps (barring uneven characters and arrangement issues, representing $90 \%$ of all vibration particular infringement). A compelling change of the bearing house is entirely adaptable to add strengthening gussets between the external dividers of the lodging and the spine of the cabinet. On account of moving houses with "pull pockets" that surpass the capacity territory, as is frequently the situation, if the trickle pockets are open over the upper part where the solidness of the lodging connection is incredibly diminished, particularly on the straight side. Also, this plan permits bearing houses to "turn over" under even stacking to the side from the pole. An allowable solution for such a circumstance is to plan a cover or a strong strengthened extension that makes a "360 degree" lodging connection, and such plan enhancements are accessible from different makers. 


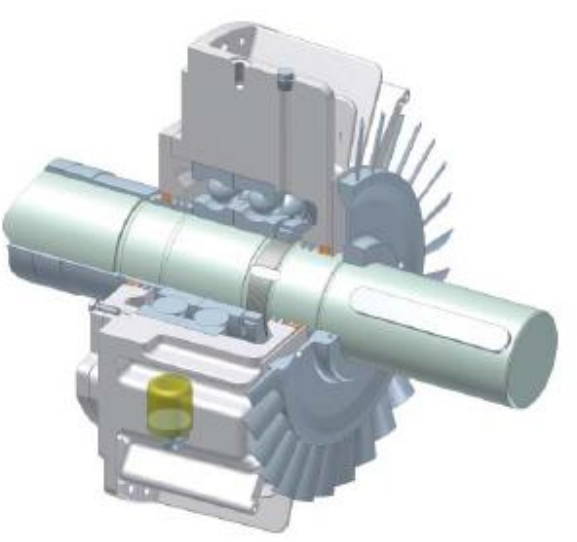

Fig 9 - Bearing Housing

\section{SHAFT OR ROTOR}

The pump shaft is intended for huge size so it works under burden with insignificant deviation. The pump shaft is the center of the pump pivoting article, and all turning parts are appended to it. Impellers and different things, for example, ornamental sleeves and push balance gadgets can be joined to the lock shaft in blend with a nearby shutting slip or with different levels of media balance. The main favorable position is that it eliminates the incense from capacity the objectives are basic, however because of the snugness of the pole, just as the chance of the pole of the pole/shaft. Inadmissible strength can prompt a change in basic speed esteems from pump to-pump of a similar kind. Stick-sneak by solid stacking during activity can cause hysteretic evacuation to the noticeable shaft connector, which if tight enough can cause rotor shakiness. Shaft vibration is frequently an intricate issue, and it is known as "rotor elements." The most exact strategies for deciding characteristic rotor waves, and the main solid approach to check the general rotor solidness or "constrained reaction" because of a specific level of disparity, is a finished rotor elements examination performed with uncommon PC frameworks. This is on the grounds that there are issues in examining the rotors contrasted with playing out a similar vertical investigation [18]. For instance, paying little mind to the kind of burden utilized on a specific pump, the response power from the course to react to vibrations and the heaps are not straight forward. In addition to the direct blocking force of each object acting directly against the rotor movement, there are other important forces acting differently in this movement, namely damping and combining "coupling forces" ("spring" reaction forces acting differently in the motion shaft), and this can be greater or greater there is a direct force, allowing the dominance to vibrate. Unlike other types of manual or computer vibration analyzes, rotor dynamic analysis incorporates the effects of these forces in motion, allowing the dependence of the rapid response speed to be modeled, and including impeller, disk balance, and aggregation gyroscopic effects.

\section{SHAFT SLEEVE}

The shaft sleeve will protect the shaft from inserting \& also protect the shaft from air pump. In addition to the pleasing fluid strength due to the action of the generators, and the effective use of the fluid in the carrier, strong hydraulic power can occur at the pump "signals of the year," that is, wear rings, interstage bushings, and measuring device clearance spaces. With this, each annular signal acts as a specific carrier, usually having a tendency to fix the rotor uphold and hoist the normal waves to higher qualities, in any event until the grant is worn. Nonetheless, as exhibited by Childs '(1982) work, the normal" firmness "can really be" debilitating "if an excess of liquid is available in the sign-manufactured region, just as different impacts, for example, the yearly" dynamic weight "and cross-blending mix ought to be incorporated. The Lomakin impact is especially solid on multistage pumps since multistage rotors are long and adaptable [17]. Since the pubic signs are chiefly situated in the center piece of the Rotor, where they utilize a great deal of power in the primary rotor twisting mode, the commitment of the help to the rotor backing can be contrasted with the firmness of the thigh itself. Shaft sleeves The sleeves are at times fitted with a key and/or lower-fitting shaft in zones where contact with metal constantly or contact and checking. This gives wear opposition, and makes the last shower change simpler and more affordable. It is significant that the sleeves are worncoordinated to the contradicting part, as shown in the gallon graphs like those in the Marscher section in the ASM Handbook (Marscher, 1993). Moreover, there should be at any rate a 50 BHN (Brinnell) hardness contrast among moving and fixed parts.

\section{COUPLING}

Bonding is used to transmit power and take out the faults of the pump and motor shaft. Inconsistent alignment when the rotating axis is different from angular alignment when the pump and motor components are not coaxial or parallel. The junction attaches the driver (e.g., Motor) shaft to the pump shaft. Pairs can be tight or flexible. Sturdy assembly requires good tolerance and assembly procedures, otherwise the drive-ups may be overloaded due to inconvenience. Flexible mixing allows for some imbalance, although vibration rises in incomplete understanding [19]. Increased vibration can be acceptable, and this can be judged by reference to various specific public vibration approvals, such as the Hydraulic Institute / ANSI standards (HI / ANSI, 2000) or API 610 (1995) standards. The general rule of thumb is that any solid combination or flexible combination with a single flexible area must be well aligned between 25 microns, and not worse than twice that number. For dynamic junctions with more than one variable point (or "area-inclusion"), the number of allowable variables depends on the speed and length of the space (e.g., distance between engagement zones). Note the other charts produced by the well-intentioned producers of the links in this case. These charts are based on how the misalignment of the joint can tolerate without self-injury, which is much more common than the pump or its driver can tolerate. Once again, refer to the public specification for guidance, both in terms of the specified high inconsistencies, and the permissible vibration rate that was improperly generated. However, the sixth general rule is that no more than $1 / 4$ to 6 to 12 microns incorrectly in inches of space should be tolerated.

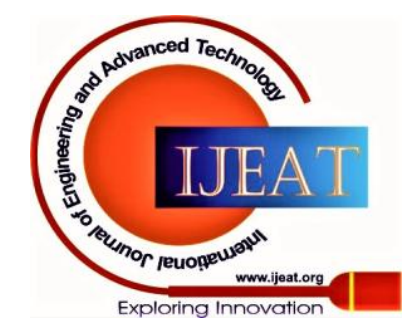




\section{Study of Mechanical Performance Affecting Factors in Split Casing Pump}

\section{BASE PLATE}

The base plate of the pump is a visible link between the feet of the bullets and the base. The base plate and the base have a certain degree of flexibility, so it is a factor in the overall rigidity when the weight of the pump is "mechanically" built into the ground. Hence, the base plate and the base are regularly the way to building up the alleged "reed" influxes of the pump, the vibrating developments that are typically communicated by the pumps standing precisely close to the running rate. This component in the establishment and preparing of new pumps is frequently neglected by development specialists and apparatus temporary workers when planning and building new or refreshed pumps. Normally, in such cases the architect or temporary worker is prepared to be worried about the low auxiliary quality adequate to help the heaviness of the hardware, however he doesn't comprehend that standing power isn't equivalent to the quality of bowing [20]. The more prominent the separation to the focal point of gravity of the pump and/or the sweep of the gyration (comparative with the movement of the reed) as indicated by the "foot" of the base plate of the pump, this impact is more huge. This is particularly valid for adaptable speed activity, where on stale pumps it is conceivable that the basic recurrence of reeds will be balanced by the by and large worthy estimations of imbalance and irregularity, working at a solitary running rate. This issue can likewise be an issue in huge, low-ascent structures with numerous sections and solid shafts to help the heaviness of hardware, yet it has solid neighborhood bowing quality in light of the fact that such bowing quality isn't needed to help the vertical pump. Notwithstanding the development of new plants, a circumstance wherein unnecessary adaptable twisting has hampered the last establishment of existing overhead water channels where existing steps are needed to take on extra pump sizes.

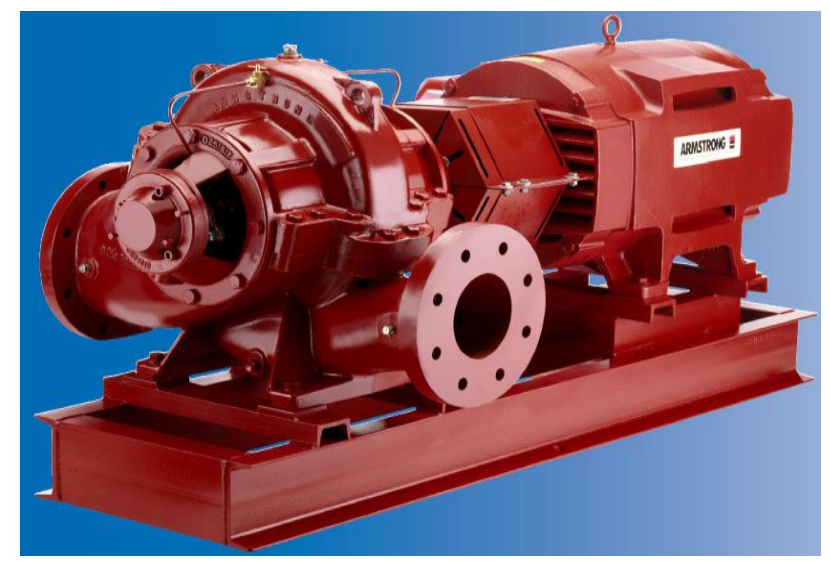

Fig 10 - split casing pump mounted on Base plate

Another important feature of basic plates and their durability involves the ability of horizontal pumps to withstand the deviation from direct or indirect planes due to force and times forced on the suction and extraction of pipes by pipes, or by pressure forces on the members to increase uninterrupted fixed power. Such distortions can create significant tension between the pump shaft and the driver's throat due to plate distortion. This can be a problem even if the pump and the motor are mounted on a standard base plate, if the base plate is too flexible and not fully held in place.

\section{CONCLUSION}

The determination for this investigation was to clarify pump thoughts, wording, development, and demonstrated critical thinking and analytic techniques so that those engaged with the determination, activity and upkeep of radiating pumps could build their viability for the benefit of the plants they use.

Despite the fact that the core of the radial pump configuration contains its own pressure driven section structure, an assortment of mechanical issues should be considered to guarantee productive and solid activity. This is particularly valid for enormous force pumps, for example, evaporator feed pumps and other cleaning pumps. Along these lines, it is significant for the individuals who compose the subtleties or settle on buying choices to comprehend the advantages of the different mechanical and innovative choices accessible to them comparable to outward pumps; the expense of plant rest periods and support costs will regularly be fundamentally influenced relying upon the variables estimated in the pump choice cycle. Also, administrators need to comprehend the expense of mechanical fix hardware by planning power, and the degree of danger they take on the off chance that they keep on working when the pump gives indications of different issues. At long last, nourishment gatherings ought to know about the ever-concealed indications of pump harm, that numerous issues and their manifestations are legitimately identified with a specific part, just as present day instruments and segments accessible for the arrangement of ongoing issues.

Mechanical issues, for example, the impacts of receiver burdens and vibration acknowledgment methodology can be effectively followed. Indeed, it requires training and information to arrive at the correct resolution in regards to the many related issues related with choosing and utilizing a divergent pump. The motivation behind this investigation is to give a "beginning stage" for the individuals who were associated with the cycle, and maybe to add an alternate point of view and a couple of business techniques for more experienced pump clients and investigators. The following are a few remarks that energize the usage of the substance introduced in this instructional exercise:

1) Inspect equipment "before," before installation, and especially before purchase. If you do not have an in-house team to do this, hire a third party consultant, or make it part of the bidding process that the manufacturer must do the analysis in a reliable way. However, there are plenty of "ballpark" checks and easy analysis that you, as an expert, can do for yourself.

2) Be careful about the size of the pump you are buying comparing what you really need with your process and its pumping system. Don't buy too many pumps that have to spend a lot of time working on a certain load.

3) Be very careful in inspecting and controlling pipe loads. Stretch joints can reduce thermal expansion, only leading to greater hydraulic lifting, making the condition worse than ideal.

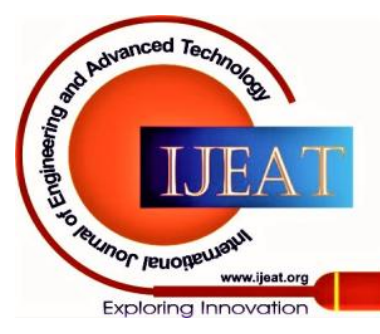


4) In the case of rotor dynamics, alignment monitoring, and natural resonance testing, the use of computer tools is more likely to lead to more accurate conclusions than traditional "hands-on" techniques.

\section{REFERENCES}

1. Ahmad Nourbakhsh "Experimental study of characteristic curves of centrifugal pumps in different specific speeds". Journal of Experimental Thermal and Fluid Science 32, 2008, pp. 800-807.

2. W.K. Chan, L.P. Chua-"The flow patterns within the impeller passages of a centrifugal pump model". Journal of Medical Engineering and Physics 22, 2000, pp. 381-393.

3. John Veness, Clifford steer and Steven Rose, inside the seal less pump, proceedings of 9th international pump users symposium, pp. 29-38 (1992).

4. Jerome A. Lorenc and Lev Nelik, Analysis \& testing of alternative bearing materials for applications in seal less pumps, proceedings of 11th international pump users symposium, pp. 51-56, (1994).

5. Donald P. Slotemen and Mark Pierecy, Developing seal less integral motor pumps using axial field, permanent magnet, disk motors, proceedings of 17th international pump users symposium, pp. 53-67, (2000).

6. James R. Brennan, Understanding and Using Sealless Rotary Pumps, Pump Users Expo '98, Cincinnati, OH, USA, (1998).

7. William R. Finley and Mark M. Hodowanec, Sleeve vs. anti-friction bearings: selection of the optimal bearing for induction motors, Paper IEEE/PCIC 2001-22, pp. 1-13, (2001).

8. Vugar A. Mammadov, Agil Yusifov and Ken P. Tacon, Upstream Pumping technology in centrifugal pump mechanical sealing applications, proceedings of 26th international pump users symposium, pp. 27-32, (2010).

9. David Clark and Alan English, The applications of Sealless magnetic drive pumps, IMechE European fluid Machinery congress, pp. 1-8, (2008).

10. Selvarasu.A, Ganthavel.K, Krishnaraj.R, Karpagarajan.S, Ravi.G and Ramesh.R, Conceptual Framework of Centrifugal Pumps towards Industrial Revolution, International Journal of Scientific \& Engineering Research, Volume 3, Issue 2, February -2012, pp. 1-7, (2012).

11. Flach P.M., Murphy M.J. and Anderson W.I., Analyzing unacceptable seal performance, proceedings of 5th international pump users symposium, Turbo machinery Laboratory , pp. 21-25, (1998).

12. Marscher, W., 1999, "Bearing Application and Lubrication"

13. Chapter, Modern Marine Engineer's Manual, Third Edition, Centreville, Maryland: Cornell Maritime Press.

14. API Standard 610, "Centrifugal Pumps for Petroleum,

15. Heavy Duty Chemical and Gas Industry Services," Eighth Edition, American Petroleum Institute, Washington, D.C., 1995.

16. ASME Boiler \& Pressure Vessel Code, American Society of Mechanical Engineers, New York, New York, 2001.

17. France, D., "Rotordynamics Considerations in the Design of High Speed Centrifugal Pumps," Proceedings of Indian Pump Manufacturers Association, 1987

18. Marscher, W. D., "Wear and Erosion of Pumps," Chapter in Volume 18 of the ASM Metals Handbook, American Society of Metals International, Materials Park, Ohio. 1993.

19. Marscher, W. D., "Vibration" Chapter and "Predictive

20. Maintenance" Chapter, STLE Tribology \& Lubrication Handbook, Society of Tribologists and Lubrication Engineers, Park Ridge, Illinois, 1997

21. Marscher, W., "Bearing Application and Lubrication"Chapter, Modern Marine Engineer's Manual, Third Edition, Centreville, Maryland: Cornell Maritime Press, 1999

22. Patel, V. P. and Coppins, D. G., "Selection of API 614, Fourth Edition, Chapter 3, General Purpose Lube Oil System Components for Rotating Process Equipment," Proceedings of the Eighteenth International Pump Users Symposium, Turbo machinery Laboratory, Texas A\&M University, College Station, Texas, ,2001, pp. 89-100.

23. Kirloskar Brothers Limited "Rotor dynamic pumps"

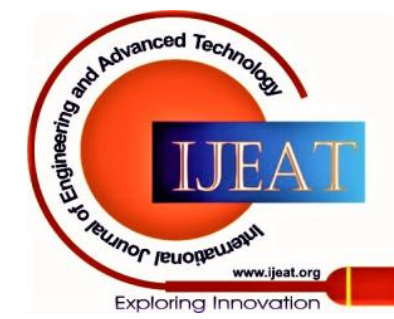

